\title{
EQUIVALENCE OF $G$-ALGEBRAS FOR ABELIAN $G$
}

\author{
ALEXANDRE TURULL
}

(Communicated by Ronald M. Solomon)

\begin{abstract}
We describe the equivalence classes of central simple $G$-algebras over an infinite field $F$ for $G$ a finite abelian group, provided the following holds. For each prime $p$ for which the field $F$ has primitive $p$ th roots, the Sylow $p$-subgroup of $G$ is either cyclic or elementary abelian.
\end{abstract}

\section{INTRODUCTION}

The description of the Schur indices of the characters of the covering groups of the symmetric and alternating groups in [2] made use of the elements of the Brauer-Wall group; see [1] for a definition of the Brauer-Wall group. The characters of these groups come in sets of three: either two characters of the covering group of the symmetric group and one of the alternating group (the former are then the two extensions of the latter), or one character of the covering group of the symmetric group and two of the alternating group (the former is then induced from each of the latter ones). In [2], it is seen how to each such triple corresponds, in a natural way, an element of the Brauer-Wall group. This element gives the following information about the triple: Whether the characters of the covering group of the symmetric group is induced or an extension of a character of the covering group of the alternating group; the field of definition of the three characters involved; and the centralizer algebra (and hence the Schur indices) of each of the three characters.

The use of the Brauer-Wall group is possible because the index between the two groups involved is two. In [3], it is shown how the results mentioned in the previous paragraph can be generalized to quotient groups of arbitrary finite order. One needs a generalization of the Brauer-Wall group. Fix some finite group $G$ (which in the applications will be the quotient group of the group by its normal subgroup) and a field $F$ of characteristic zero. First one defines what is meant by a central simple $G$-algebra $A$. Simple means, of course, that it has exactly two $G$-invariant two-sided ideals. Central here means that $\mathrm{C}_{\mathrm{Z}(A)}(G)=F$. One then defines which, among those, are trivial $G$-algebras. These are the $G$-algebras $E$ which are just the full $F$-endomorphism algebra

Received by the editors September 25, 1993.

1991 Mathematics Subject Classification. Primary 20C15.

Key words and phrases. Brauer group, $G$-algebra, finite groups, representations.

Partially supported by grants from the NSA and the NSF. 
of a nonzero $F G$-module, with the natural action of $G$ on $E$. We say that two central simple $G$-algebras $A$ and $B$ are equivalent if there exist trivial $G$-algebras $E$ and $E^{\prime}$ such that

$$
A \otimes E \simeq B \otimes E^{\prime}
$$

as $G$-algebras, where the tensor products are over $F$. This is an equivalence relation, and the set of equivalence classes is denoted by $S(G, F)$. In the case where $G=1$, this is just the set of elements of the Brauer group of $F$. In the case where $|G|=2$, this can be seen to be equivalent to the set of elements of the Brauer-Wall group.

Let $\Gamma_{1}$ be a finite group and $\Gamma_{2}$ be a normal subgroup of $\Gamma_{1}$ such that $\Gamma_{1} / \Gamma_{2} \simeq G$. Then, to each irreducible character $\chi$ of $\Gamma_{1}$ corresponds, in a natural way, an element $[[\chi]]$ of $S\left(G, \mathbb{Q}\left(\chi_{\mid \Gamma_{2}}\right)\right)$; see [3] for details. The element $[[\chi]]$ contains in it the information about the operations of induction and restriction among the characters of the subgroups of $\Gamma_{1}$ that contain $\Gamma_{2}$, their relative degrees, their fields of definition, and their centralizer algebras (and hence their Schur indices); see [3] for details. The study of Clifford theory with Schur indices can then be decomposed into two problems. One is to describe the equivalence classes of central simple $G$-algebras. The other is to assign, to each particular character and normal subgroup, the corresponding class of central simple $G$-algebras.

Hence, one would like, in particular, to effectively describe the equivalence classes of $G$-algebras for each finite group $G$. In [4], we define for each central simple $G$-algebra $A$ an inertia subgroup $I$ of $G$. This subgroup is defined up to conjugacy in $G$ and depends only on the equivalence class of $A$. Furthermore, we define the $I$-centroid $\Delta(A, I)$ (or simply $\Delta(A)$ if $I$ is normal in $G$ ) of $A$, which is a central fully $I$-graded $\mathrm{N}_{G}(I)$-algebra over $F$ of dimension $\left|\mathrm{N}_{G}(I)\right|$, and depends also only on the equivalence class of $A$. See $\S 1.1$ below for the definition of a central fully $I$-graded $\mathrm{N}_{G}(I)$-algebra over $F$. In [5], it is shown how to characterize the equivalence classes of central simple $G$-algebras $A$ which have a fixed centroid, provided the inertia group is normal in $G$ and the centroid has a special property, namely being complemented; see Definition 1.2.

In the present paper, we show that, provided $G$ is abelian and for each prime $p$ for which the field contains a primitive $p$-root of 1 , the Sylow $p$-subgroup of $G$ is either elementary abelian or cyclic, then the results of [5] suffice to characterize each equivalence class of central simple $G$-algebras. Suppose $F$ is an infinite field and $G$ is such an abelian group. Let $I$ be a subgroup of $G$. We show that these conditions imply that every central fully $I$-graded $G$-algebra (see Definition 1.1 below) will have some complement $R$ (see Definition 1.2 below). Now the central fully $I$-graded $G$-algebras $\Delta$ over $F$ which admit the complement $R$ are classified, up to isomorphism, by $\Delta_{1}$ as a $G$-algebra and the invariant $\eta(\Delta, R) \in \mathrm{H}^{2}\left(I, F^{\times}\right)$; see Theorem 2.4 in [5] for a more precise statement. Furthermore, one can describe the equivalence classes of central simple $G$-algebras with $I$-centroid $\Delta$ as follows.

Theorem. Let $G$ be a finite abelian group and $F$ an infinite field such that, for each prime $p$ for which $F$ has a primitive pth root of 1 , the Sylow p-subgroup of $G$ is either cyclic or elementary abelian. Fix $a \Delta$ as above. Then the equivalence classes of central simple G-algebras whose inertia group is $I$ and for which 
$\Delta(A, I) \simeq \Delta$ are in one-to-one correspondence with the cosets $\operatorname{Br}(F) / \operatorname{Sbr}\left(R, \Delta_{1}\right)$. Here $\operatorname{Br}(F)$ is the Brauer group of the field $F$ and $\operatorname{Sbr}\left(R, \Delta_{1}\right)$ is a finite subgroup of $\operatorname{Br}(F)$ which depends only on $\Delta_{1}$ and the action of $R$ on it; see Definition 4.8 in [5].

Proof. Since the centroid has a complement (see Theorem 2.7 below), the theorem follows directly from Theorem 5.3 in [5] (see also Theorem 1.3 below). We refer the reader to [5] for further details.

In particular, the theorem classifies all the classes of central simple $G$-algebras over $F$, whenever $F$ is a real field and $G$ is a abelian group with either cyclic or elementary abelian Sylow 2-subgroup.

\section{SOME DEFINITIONS AND RESULTS}

Throughout, $F$ will denote an infinite field and $G$ a finite group. We begin by recalling some of the basic definitions and results from [4] and [5]. We refer the reader to these papers for further notation.

Definition 1.1. Let $F$ be an infinite field, $H$ a finite group, and $I$ a normal subgroup of $H$. A structure $\Delta$ is said to be a central fully I-graded $H$-algebra over $F$ if the following hold.

(a) $\Delta$ is a fully $I$-graded algebra over $F$, that is, we are given

$$
\Delta=\bigoplus_{g \in I} \Delta_{g}
$$

where this is a direct sum decomposition of vector spaces over $F$, and the algebra structure of $\Delta$ is such that $\Delta_{g} \Delta_{h}=\Delta_{g h}$ as sets for all $g, h \in I$.

(b) We are also given an action of $H$ on $\Delta$ in such a way that the action preserves the algebra structure of $\Delta$, for every $h \in I$ and $a \in \Delta_{h}, b \in \Delta$, we have $a b={ }^{h} b a$, and for all $h \in H, g \in I, a \in \Delta_{g}$, and we have ${ }^{h} a \in \Delta_{h g h-1}$.

(c) Finally, $\Delta_{1}$ is a finite-dimensional semisimple commutative algebra, the centralizer of the action of $H$ on $\Delta_{1}$ is $I$ and $\mathrm{C}_{\Delta_{1}}(H)=F .1=F$.

Definition 1.2. Let $I$ be a normal subgroup of $G$. Let $\Delta$ be a central fully $I$-graded $G$-algebra. A complement to $\Delta$ is any subgroup $R$ of $G$ such that $R \subseteq \mathrm{Z}(G), R \cap I$ acts trivially on $\Delta$, and $G=I R$. We denote by $\kappa(\Delta)$ the set of all complements to $\Delta$. We say that $\Delta$ is complemented if $\kappa(\Delta) \neq \varnothing$.

Theorem 1.3. Let $F$ be some infinite field. Let $G$ be a finite group, $I$ and $R$ normal subgroups of $G$ such that $G=I R$ and $R \subseteq \mathrm{Z}(G)$. Let $\Delta$ be a central fully I-graded G-algebra such that $R \in \kappa(\Delta)$. Set $\eta=\eta(\Delta, R) \in$ $\mathrm{H}^{2}\left(I, F^{\times}\right)$. Pick any $\xi \in \Xi(\eta, R)$, which exists by Lemma 3.2 in [5]. Then, the equivalence classes of central simple $G$-algebras $A$ such that $A$ has inertia group $I$ and $\Delta(A) \simeq \Delta$ are in one-to-one correspondence with the elements of $\operatorname{Br}(F) / \operatorname{Sbr}\left(R, \Delta_{1}\right)$ via the map

$$
A \rightarrow \mathfrak{b}(A, R, \xi) .
$$

Proof. This is just Theorem 5.3 in [5]; see [5] for the unexplained notation.

\section{A BILINEAR FORM}

Suppose now that $G$ is a finite abelian group and $\Delta$ is a central fully $I$ graded $G$-algebra over the infinite field $F$. From $\Delta$ one can easily obtain a bimultiplicative symplectic form on $I$. 
Definition 2.1. We define the map

$$
\beta_{\Delta}: I \times I \rightarrow F^{\times}
$$

as follows. If $g, h \in I$ let $t_{g} \in \Delta_{g}$ and $t_{h} \in \Delta_{h}$ be any invertible elements. We set

$$
\beta(g, h)=t_{g} t_{h} t_{g}^{-1} t_{h}^{-1} \text {. }
$$

Proposition 2.2. Let $\Delta$ be a central fully I-graded G-algebra. Then $\beta_{\Delta}$ is well defined and bimultiplicative and satisfies

$$
\beta_{\Delta}(h, h)=1
$$

for all $h \in I$.

Proof. Since $\Delta$ is a fully $I$-graded algebra, there exist invertible $t_{g}$ and $t_{h}$. It follows that

$$
t_{g} t_{h} t_{g}^{-1} t_{h}^{-1} \in \Delta_{1}^{\times}
$$

Let $t_{g}^{\prime} \in \Delta_{g}$ and $t_{h}^{\prime} \in \Delta_{h}$ be invertible in $\Delta$. Then there exist $z, z^{\prime} \in \Delta_{1}^{\times}$such that $t_{g}^{\prime}=t_{g} z$ and $t_{h}^{\prime}=t_{h} z^{\prime}$. Hence,

$$
t_{g}^{\prime} t_{h}^{\prime}\left(t_{g}^{\prime}\right)^{-1}\left(t_{h}^{\prime}\right)^{-1}=t_{g} t_{h} t_{g}^{-1} t_{h}^{-1}
$$

so that $\beta_{\Delta}(g, h)$ is a uniquely defined element of $\Delta_{1}^{\times}$. Since $G$ acts on $\Delta$ stabilizing both the sets $\Delta_{g}$ and $\Delta_{h}$, for every $x \in G$, we have

$$
{ }^{x} \beta_{\Delta}(g, h)=\beta_{\Delta}(g, h) .
$$

Hence, $\beta_{\Delta}(g, h) \in \mathrm{C}_{\Delta_{1}}(G)^{\times}=F^{\times}$, as $\Delta$ is a central fully $I$-graded $G$-algebra. Hence $\beta_{\Delta}$ is well defined.

Let $g, h, h^{\prime} \in I$ and pick $t_{g} \in \Delta_{g}, t_{h} \in \Delta_{h}$, and $t_{k^{\prime}} \in \Delta_{h^{\prime}}$ all invertible. Then

$$
\beta_{\Delta}\left(g, h h^{\prime}\right)={ }^{t_{g}}\left(t_{h} t_{h^{\prime}}\right)\left(t_{h} t_{h^{\prime}}\right)^{-1}={ }^{t_{g}} t_{h} \beta_{\Delta}\left(g, h^{\prime}\right) t_{h}^{-1}=\beta_{\Delta}(g, h) \beta_{\Delta}\left(g, h^{\prime}\right),
$$

as $\beta_{\Delta}\left(g, h^{\prime}\right) \in \mathrm{Z}(\Delta)$. Hence $\beta_{\Delta}$ is multiplicative in the second variable. A similar argument shows $\beta_{\Delta}$ is bimultiplicative. It follows immediately from the definition that $\beta_{\Delta}(h, h)=1$ for all $h \in I$. This completes the proof of the proposition.

Definition 2.3. Let $\Delta$ be a central fully $I$-graded $G$-algebra. We set

$$
\beta_{\Delta}^{\perp}=\left\{h \in I: \text { for every } g \in I, \beta_{\Delta}(h, g)=1\right\} \text {. }
$$

Proposition 2.4. Let $\Delta$ be a fully I-graded central $G$-algebra. Then $\beta_{\Delta}^{\perp}$ is $a$ subgroup of I. Furthermore, whenever $p$ is a prime and $p$ divides the order of the quotient group $I / \beta_{\Delta}^{\perp}$, then $F$ has a primitive pth root of 1 and the Sylow p-subgroup of $I / \beta_{\Delta}^{\perp}$ is not cyclic.

Proof. That $\beta_{\Delta}^{\perp}$ is a subgroup of $I$ follows immediately from the fact that $\beta_{\Delta}$ is bimultiplicative. Suppose $p$ is a prime and $p|| I / \beta_{\Delta}^{\perp} \mid$. If $g, h \in I$, since $\beta_{\Delta}$ is bimultiplicative and $\beta_{\Delta}(g h, g h)=\beta_{\Delta}(g, g)=\beta_{\Delta}(h, h)=1$, we have $1=\beta_{\Delta}(g, h) \beta_{\Delta}(h, g)$ so that

$$
\beta_{\Delta}(h, g)=\beta_{\Delta}(g, h)^{-1}
$$


holds. Hence, whenever $g, h \in I$ and $x, y \in \beta_{\Delta}^{\perp}$ we have

$$
\beta_{\Delta}(g x, h y)=\beta_{\Delta}(g, h)
$$

since, by the definition of $\beta_{\Delta}^{\perp}$ and the above, $\beta_{\Delta}(x, h)=\beta_{\Delta}(g, y)=\beta_{\Delta}(x, y)$ $=1$. Set $\bar{I}=I / \beta_{\Delta}^{\perp}$. It follows that we may define a map

$$
\bar{\beta}_{\Delta}: \bar{I} \times \bar{I} \rightarrow F^{\times}
$$

by $\bar{\beta}_{\Delta}\left(g \beta_{\Delta}^{\perp}, h \beta_{\Delta}^{\perp}\right)=\beta_{\Delta}(g, h)$. Then $\bar{\beta}_{\Delta}$ is bimultiplicative. By Cauchy's Theorem, there exists some element $\bar{h} \in \bar{I}$ of order $p$. Since $\bar{h} \neq 1$, there exists some $\bar{g} \in \bar{I}$ such that $\bar{\beta}_{\Delta}(\bar{h}, \bar{g}) \neq 1$. Since $\bar{\beta}_{\Delta}$ is bimultiplicative , $\bar{\beta}_{\Delta}(\bar{h}, \bar{g})^{p}=\bar{\beta}_{\Delta}(\overline{1}, \bar{g})=1$. Hence $\bar{\beta}_{\Delta}(\bar{h}, \bar{g})$ is a primitive $p$ th root of 1 and an element of $F$.

Suppose, by way of contradiction, that the subgroup $\langle\bar{h}, \bar{g}\rangle$ of $\bar{I}$ generated by $\bar{h}$ and $\bar{g}$ is cyclic. Say $\langle\bar{h}, \bar{g}\rangle=\langle\bar{a}\rangle$. Then, by Proposition 2.2,

$$
\bar{\beta}_{\Delta}(\bar{a}, \bar{a})=1,
$$

and, since $\bar{\beta}_{\Delta}$ is bimultiplicative and both $\bar{h}$ and $\bar{g}$ are powers of $\bar{a}$, it follows that

$$
\bar{\beta}_{\Delta}(\bar{h}, \bar{g})=1,
$$

a contradiction. Hence $\langle\bar{h}, \bar{g}\rangle$ is not cyclic. Since $\bar{I}$ is finite abelian and $\bar{h}$ has order $p$, this implies that $\langle\bar{h}, \bar{g}\rangle$ does not have a cyclic Sylow $p$-subgroup. Hence the Sylow $p$-subgroup of $\bar{I}$ is not cyclic either. This concludes the proof of the proposition.

Corollary 2.5. Suppose $\Delta$ is a central fully I-graded G-algebra. If, for every prime $p$ for which $F$ has a primitive pth root of $1, I$ has a cyclic Sylow $p$ subgroup, then $\beta_{\Delta}^{\perp}=I$. In particular, this will be the case if $F=\mathbb{Q}$ and I has a cyclic Sylow 2-subgroup.

Proof. This follows immediately from Proposition 2.4.

Proposition 2.6. Suppose $\Delta$ is a central fully I-graded G-algebra. Then $\beta_{\Delta}^{\perp}$ is the group of gradings of $\mathrm{Z}(\Delta)$. Furthermore, as a subgroup of $G, \beta_{\Delta}^{\perp}$ acts trivially on $\Delta$.

Proof. For each $h \in I$, choose some $t_{h} \in \Delta_{h}$ which is invertible in $\Delta$. Then $\Delta$ is generated, as an algebra, by $\Delta_{1}$ and $\left\{t_{h}: h \in I\right\}$. By Definition 1.1(b), $\Delta_{1}$ is in the center of $\Delta$. The center $Z(\Delta)$ of $\Delta$ is a graded subalgebra of $\Delta$ generated by $\Delta_{1}$ and

$$
\left\{t_{h}: t_{h} \text { commutes with } t_{g} \text { for all } g \in G\right\} \text {. }
$$

Hence, it follows from Definitions 2.1 and 2.3 that the group of gradings of $\mathrm{Z}(\Delta)$ is exactly $\beta_{\Delta}^{\perp}$. Let $h \in \beta_{\Delta}^{\perp}$. Pick any element in $\Delta$, say $a \in \Delta$. By Definition 1.1(b), we have

$$
t_{h} a t_{h}^{-1}={ }^{h} a \text {. }
$$

Since $t_{h} \in Z(\Delta)$, it follows that ${ }^{h} a=a$ for every homogeneous element $a \in \Delta$. Hence, as a subgroup of $G, \beta_{\Delta}^{\perp}$ acts trivially on $\Delta$. This completes the proof of the proposition. 
Theorem 2.7. Let $\Delta$ be a central fully I-graded G-algebra, where $G$ is an abelian group. Then, if for every prime $p$ for which $F$ contains a primitive pth root of $1, G$ has either a cyclic or elementary abelian Sylow p-subgroup, then $\kappa(\Delta) \neq \varnothing$. If, for every $p$ for which $F$ contains a primitive pth root of 1 , $G$ has a cyclic Sylow p-subgroup, then $\beta_{\Delta}^{\perp}=I$.

Proof. Suppose that for every prime $p$ for which $F$ has a primitive $p$ th root of $1, G$ has either a cyclic or an elementary abelian Sylow $p$-subgroup. Let $\pi$ be the set of all primes $p$ dividing $\left|I / \beta_{\Delta}^{\perp}\right|$. Let $H$ be the Hall $\pi$-subgroup of $G$, and $H^{\prime}$ be the Hall $\pi^{\prime}$-subgroup of $G$. By Proposition 2.4, for each $p \in \pi$, $F$ has a primitive $p$ th root of 1 and the Sylow $p$-subgroup of $G$ is not cyclic. By hypothesis, it follows that the Sylow $p$-subgroup of $G$ is then elementary abelian. Hence, $H$ is a direct product of elementary abelian groups. Therefore $I \cap H$ has a complement in $H$, so there is some subgroup $C$ of $H$ such that $C(I \cap H)=H$ and $C \cap I=1$.

Set $R=C H^{\prime}$. Then $R I \supseteq H H^{\prime}=G$, so $R I=G$. Suppose $p$ is a prime and $p \mid\left[R \cap I: R \cap \beta_{\Delta}^{\perp}\right]$. Let $P$ be the Sylow $p$-subgroup of $G$. Then $P \cap S$ is the Sylow $p$-subgroup of $S$, for every subgroup $S$ of $G$. Hence, $p \mid[P \cap R \cap$ $\left.I: P \cap R \cap \beta_{\Delta}^{\perp}\right]$. Since $P \cap R \cap I / P \cap R \cap \beta_{\Delta}^{\perp}$ is isomorphic to a subgroup of $P \cap I / P \cap \beta_{\Delta}^{\perp}, p \in \pi$. By construction, it then follows that $P \cap R=P \cap C$ and $P \cap R \cap I=P \cap C \cap I=1$, a contradiction. Hence, no such $p$ exists and $R \cap I \subseteq \beta_{\Delta}^{\perp}$. By Proposition 2.6, $R \cap I$ acts trivially on $\Delta$. Hence, by Definition 1.2, $R \in \kappa(\Delta)$ and $\kappa(\Delta) \neq \varnothing$, as desired. That $I=\beta_{\Delta}^{\perp}$ whenever for every $p$ for which $F$ contains a primitive $p$ th root of $1, G$ has a cyclic Sylow $p$-subgroup, follows immediately from Corollary 2.5 . This completes the proof of the theorem.

\section{REFERENCES}

1. T. Y. Lam, The algebraic theory of quadratic forms, Benjamin, Reading, MA, 1973.

2. A. Turull, The Schur index of projective characters of symmetric and alternating groups, Ann. of Math. (2) 135 (1992), 91-124.

3. __ Clifford theory with Schur indices, J. Algebra (to appear).

4. __ Some invariants for equivalent G-algebras, J. Pure Appl. Algebra (to appear).

5. __ Equivalence of G-algebras with complemented centroid, Comm. Algebra 22 (1994), 5037-5078.

Department of Mathematics, University of Florida, Gainesville, Florida 32611

E-mail address: turull@math.ufl.edu 